\title{
CÁLCULOS DOS COEFICIENTES DE TRANSFERÊNCIA DE MASSA E NÚMERO DE ESTÁGIOS TEÓRICOS DE EQUIPAMENTO CONTÍNUO APLICADO AO FRACIONAMENTO DE ÓLEO ESSENCIAL CÍTRICO
}

\author{
D. GONÇALVES ${ }^{1}$, C. C. KOSHIMA ${ }^{1}$, M. E. E. TESCHKE ${ }^{1}$, C. E. C. RODRIGUES ${ }^{1}$ \\ ${ }^{1}$ Universidade de São Paulo, Faculdade de Zootecnia e Engenharia de Alimentos, Departamento de \\ Engenharia de Alimentos, Laboratório de Engenharia de Separações \\ E-mail para contato: danielg@usp.br
}

\begin{abstract}
RESUMO - O fracionamento de óleos essenciais cítricos é realizado com o intuito de melhorar as características sensoriais e estabilidade da matéria-prima. Usualmente o processo é feito por destilação simples, com emprego de altas temperaturas, o que ocasiona degradação de compostos termossensíveis e alto gasto energético. Neste contexto, a extração por solvente destaca-se como um processo alternativo. $\mathrm{O}$ dimensionamento do equipamento Perforated Rotating Disc Contactor (PRDC) utilizado para a desterpenação da mistura limoneno + linalol foi realizado pelo cálculo do número de estágios teóricos $(\mathrm{N})$ e coeficiente global volumétrico de transferência de massa de linalol $\left(\mathrm{K}_{\mathrm{R}} \mathrm{a}\right)$, em função de algumas variáveis de processo. Maiores teores de água no solvente apresentaram menores valores de $K_{R}$ a. Para baixos valores da razão $S / F$ não foi possível calcular $\mathrm{N}$ e o aumento da velocidade de rotação dos discos acarretou na diminuição do número de estágios teóricos.
\end{abstract}

\section{INTRODUÇÃO}

Óleos essenciais são importantes matérias-primas nas indústrias de alimentos e química, sendo utilizados principalmente como compostos aromatizantes e na composição de sabões, detergentes, cremes, loções, perfumes, etc. (CHÁFER et al., 2005; ARCE et al., 2005). São compostos por centenas de componentes, sendo basicamente divididos em hidrocarbonetos e seus derivados oxigenados. Os compostos oxigenados são geralmente considerados preferíveis pelo odor e, devido este fato, seu conteúdo se tornou um parâmetro crucial no valor de mercado do óleo essencial, representando maior estabilidade e melhor qualidade sensorial (GONÇALVES et al., 2014).

O fracionamento de óleos essenciais em escala industrial é efetuado com o intuito de concentrar porções dos compostos oxigenados em proporções muito maiores e, em alguns casos, concentrar alguns constituintes do óleo para um estado puro (SCHMIDT, 2010). A concentração dos compostos oxigenados é realizada por meio da retirada parcial ou total dos hidrocarbonetos (LAWRENCE, 1995).

Diferentes configurações de colunas de fracionamento são conhecidas, sendo que em 

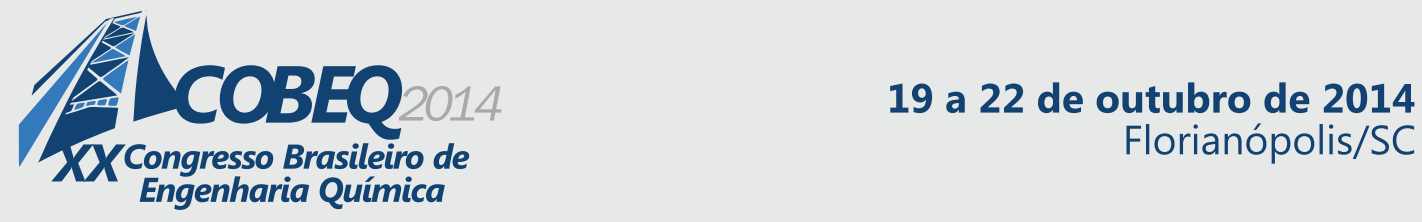

laboratórios e indústrias de pequeno porte restringe-se principalmente a colunas de vidro, aço inoxidável ou outro material inerte ao óleo essencial (SCHMIDT, 2010). Neste trabalho foi proposta a utilização de equipamento conhecido como Perforated Rotating Disc Contactor (PRDC), ou Coluna de Discos Rotativos Perfurados, que consiste em um cilindro vertical equipado com discos perfurados presos a um eixo central ligado a um motor de velocidade variável, utilizado para promover dispersão e maior contato entre o óleo e o solvente. A alimentação de óleo e solvente é realizada continuamente e em sentido contracorrente e a retirada das amostras das correntes de saída depende da densidade das mesmas (PINA, 2001).

O caminho mais utilizado para o projeto de colunas de extração operando de modo contínuo e em contracorrente é através do cálculo do número de estágios teóricos necessários para que se atinja certa concentração de componente extraído (TREYBAL, 1963). Pelas equações apresentadas por Treybal $(1963,1980)$, observa-se que o coeficiente de transferência de massa do soluto possui grande importância e relaciona-se inversamente ao número de estágios teóricos da coluna, sendo que quanto maior seu valor, menor é o número de estágios teóricos do equipamento e, consequentemente, menor é a altura equivalente da coluna.

Desta forma, por meio deste trabalho objetiva-se determinar dados que possibilitem dimensionar um equipamento contínuo PRDC aplicado ao fracionamento da mistura limoneno + linalol (óleo essencial cítrico modelo), utilizando etanol e água (em diferentes proporções) como solvente. Os dados experimentais consistiram no cálculo do número de estágios teóricos da PRDC e do coeficiente global volumétrico de transferência de massa do soluto (linalol).

\section{MATERIAIS E MÉTODOS}

\subsection{Materiais}

Para a preparação da mistura modelo de óleo essencial cítrico foram utilizadas massas conhecidas de limoneno (Sigma-Aldrich, 97-98\%, CAS 5989-27-5, EUA) e linalol (Sigma-Aldrich, 97\%, CAS 78-70-6, EUA) em três proporções (5, 10 e $15 \%$ de linalol, em massa). O solvente hidroalcoólico foi preparado por meio da adição de água ultra pura (Millipore, modelo 3UV, França) ao etanol absoluto (Merck, 97,8\%, CAS 64-17-5, Alemanha), em duas proporções (30 e $40 \%$ de água, em massa).

\subsection{Métodos}

Experimentos de extração em Coluna de Discos Rotativos Perfurados (PRDC): Foram realizados diversos experimentos de fracionamento da mistura modelo, nos quais se variaram a velocidade de rotação dos discos $(150,200$ e $250 \mathrm{rpm})$, o teor de linalol na alimentação $(5,10$ e $15 \%$, em massa) e razão entre as vazões mássicas das correntes de solvente e alimentação $(0,5 ; 1,25 ; 2,5$ e 3,0). Para os experimentos variando-se a velocidade de rotação dos discos utilizou-se alimentação com $5 \%$ de linalol (valor nominal, em massa) e razão S/F igual a 1,25 (valor nominal). Para os experimentos nos quais foi avaliada a influência da composição da alimentação adotou-se velocidade de rotação dos discos igual a $150 \mathrm{rpm}$ e razão $\mathrm{S} / \mathrm{F}$ igual a 1,25 (valor nominal). Já para os 
experimentos variando-se a razão S/F, foi utilizado alimentação com $5 \%$ de linalol (valor nominal, em massa) e velocidade de rotação dos discos igual a $150 \mathrm{rpm}$. Em todos os experimentos o solvente foi estabelecido como fase contínua e a alimentação (óleo essencial) foi estabelecida como fase dispersa. $\mathrm{O}$ equipamento operou de forma contínua e em contracorrente à temperatura controlada de $25,0 \pm 0,1{ }^{\circ} \mathrm{C}$ e pressão ambiente.

O equipamento consistiu em quatro correntes, sendo duas de entrada e duas de saída. As correntes de entrada foram: alimentação (F), ou óleo essencial e solvente (S); já as correntes de saída foram: extrato (E), caracterizada por ser rica em solvente e compostos extraídos da alimentação e rafinado (R), caracterizado por ser rico em óleo essencial e uma pequena porção de solvente. Devido à densidade das fases, a alimentação (menos densa que o solvente) foi inserida na região inferior da coluna e a corrente de extrato, por sua vez, foi retirada na região inferior.

Determinação do teor de etanol, limoneno e linalol por CG-DIC: A composição das correntes rafinado e extrato, provenientes dos experimentos de extração na coluna PRDC, foram analisadas por cromatografia gasosa com detector de ionização de chama (CG-DIC). A quantificação de etanol, limoneno e linalol foi realizada por meio de curvas de calibração construídas para cada componente. As condições experimentais utilizadas foram: coluna DBFFAP $0,25 \mu \mathrm{m}, 30 \mathrm{~m}$ x $0,25 \mathrm{~mm}$ d.i. (Agilent, EUA); hélio como gás de arraste na taxa de $1,13 \mathrm{~mL} / \mathrm{min}$; temperatura do injetor de $250{ }^{\circ} \mathrm{C}$; temperatura da coluna de $(100$ a 240$){ }^{\circ} \mathrm{C}$ (taxa de $\left.8{ }^{\circ} \mathrm{C} / \mathrm{min}\right)$; temperatura do detector de $280{ }^{\circ} \mathrm{C}$. Todas as quantificações foram realizadas no mínimo em duplicata e utilizou-se o 1-propanol como solvente cromatográfico (GONÇALVES et al., 2014).

Determinação do Teor de água: O teor de água no solvente, etanol, limoneno e linalol, bem como nas fases provenientes do equipamento PRDC foi determinado pelo método de titulação Karl Fischer. Amostras de 0,01 a 0,1 g foram tituladas com solução de Karl Fischer (KF) utilizando-se como solvente metanol/clorofórmio na proporção 4:1, em volume. O título do reagente KF utilizado foi determinado no início de cada titulação e a porcentagem mássica de água nas amostras foi fornecida automaticamente pelo aparelho.

\subsection{Cálculos}

Número de estágios teóricos da PRDC $(\mathrm{N})$ : O cálculo do número de estágios teóricos da PRDC, operando em regime permanente e em contracorrente, foi realizado de acordo com a Equação 1, segundo sugerido por Treybal (1980).

$$
\begin{aligned}
& N=\int_{w_{2, R}}^{w_{2, F}} \frac{d w_{2, R}}{w_{2, R}-w_{2, R}{ }^{*}}+\frac{1}{2} \ln \frac{1-w_{2, R}}{1-w_{2, F}}+\frac{1}{2} \ln \frac{w_{2, R}(r-1)+1}{w_{2, F}(r-1)+1}, \\
& r=\frac{M M_{1}}{M M_{2}}
\end{aligned}
$$


Onde $w_{2, R}$ é a fração mássica de linalol no rafinado, $w_{2, R}$ * é a fração mássica de linalol na condição de equilíbrio, $w_{2, F}$ é a fração mássica de linalol na alimentação, $M M$ é a massa molar do composto em questão $[\mathrm{g} / \mathrm{mol}]$.

Para o cálculo do termo integral, o intervalo entre $w_{2, F}$ e $w_{2, R}$ foi dividido em pequenos intervalos $\left(w_{2, R i}\right)$ de forma que um diagrama relacionando $1 /\left(w_{2, R i}-w_{2, R i} *\right)$ em função de $w_{2, R i}$ fosse obtido. A equação correspondente ao diagrama foi, então, integrada utilizando o software OriginLab® versão 9.0 nos intervalos de $w_{2, R}$ e $w_{2, F}$, segundo mostrado na Equação 1.

As frações mássicas de linalol no equilíbrio $\left(w_{2, R} *\right)$ foram obtidas por meio das curvas de equilíbrio do linalol, relacionando a fração mássica de linalol na corrente extrato $\left(w_{2, E}\right)$ com sua correspondente fração mássica na fase terpênica (ou rafinado). Os dados de equilíbrio líquido-líquido disponíveis no trabalho de Gonçalves (2013) foram utilizados para ajuste polinomial, a partir do qual as equações para o sistema com solvente contendo $30 \%$ de água (Equação 3) e para o solvente contendo $40 \%$ de água (Equação 4) foram obtidas.

$$
\begin{array}{ll}
w_{2, F S}=-1,3917 \cdot w_{2, F T}{ }^{2}+0,7490 \cdot w_{2, F T} & \mathrm{R}^{2}=0,9973 \\
w_{2, F S}=-0,7968 \cdot w_{2, F T}{ }^{2}+0,3642 \cdot w_{2, F T} & \mathrm{R}^{2}=0,9929
\end{array}
$$

Coeficiente global volumétrico de transferência de massa do soluto $\left(K_{R} \underline{a}\right.$ ): Os valores de $K_{R} a$ foram calculados conforme mostrado na Equação 5.

$$
R^{\prime} \times\left(w_{2, F}^{\prime}-w_{2, R}^{\prime}\right)=K_{R} \times a \times V \times \Delta w_{R, 2 M}^{\prime}
$$

Onde $\mathrm{R}^{\prime}$ é a vazão mássica da fase dispersa (rafinado) em base livre de linalol [g/min], $\mathrm{w}^{\prime}{ }_{2, \mathrm{~F}}$ é a fração mássica de linalol na alimentação em base livre de linalol, $\mathrm{w}^{\prime}{ }_{2, \mathrm{R}}$ é a fração mássica de linalol no rafinado em base livre de linalol, $K_{R} \times a\left(\right.$ ou $\left.K_{R} a\right)$ é o coeficiente global volumétrico de transferência de massa [s-1], sendo que $K_{R}$ é o coeficiente global de transferência de massa [kg de linalol/ $\left(\mathrm{m}^{2} . \mathrm{s} .(\mathrm{kg}\right.$ de linalol/ $\mathrm{kg}$ de fase rafinado))] e a é a área de transferência de massa por unidade de volume da região de extração $\left[\mathrm{m}^{2} / \mathrm{m}^{3}\right]$. V é o volume da região de extração $\left[\mathrm{m}^{3}\right]$ e $\Delta \mathrm{w}_{\mathrm{R}, 2 \mathrm{M}}^{\prime}$ é a média logarítmica das diferenças de concentração nos extremos da coluna, baseada nas unidades de concentração da fase rafinado, em base livre de linalol, calculada pela Equação 6.

$$
\Delta w_{R, 2 M}^{\prime}=\frac{\Delta w_{A}-\Delta w_{B}}{\ln \left(\frac{\Delta w_{A}}{\Delta w_{B}}\right)}
$$

Na qual:

$$
\begin{aligned}
& \Delta w_{A}=\text { diferença absoluta entre } w_{2, F}^{\prime} \text { e } w_{2, R}^{\prime}{ }^{*} \\
& \Delta w_{B}=\text { diferença absoluta entre } w_{2, R}^{\prime} \text { e } w_{2, S}^{\prime} .
\end{aligned}
$$


O termo da Equação 4 foi calculado através da relação entre as concentrações de equilíbrio após a elaboração da curva de equilíbrio. $\mathrm{w}^{\prime}{ }_{2, \mathrm{~F}}$ e $\mathrm{w}^{\prime}{ }_{2, \mathrm{R}}$ foram obtidos a partir das composições das correntes dos experimentos de extração em PRDC.

Assim como na Equação 1, o termo $\mathrm{w}^{\prime}{ }_{2, \mathrm{R}}{ }^{*}$ refere-se a fração mássica de linalol na fase rafinado no equilíbrio correspondente à $\mathrm{w}_{2, \mathrm{E}}^{\prime}$ (fração mássica de linalol na fase extrato). $\mathrm{O}$ termo $\mathrm{w}^{\prime}{ }_{2, \mathrm{~S}}$ corresponde à fração mássica de linalol no solvente. Como o solvente foi composto por etanol e água, este valor é igual a zero. O sobrescrito ' corresponde ao valor da fração mássica em base livre do soluto (linalol).

\section{RESULTADOS E DISCUSSÃO}

A Figura 1 apresenta os valores calculados do número de estágios da coluna PRDC (N) e do coeficiente global volumétrico de transferência de massa do linalol $\left(K_{R} a\right)$ em função das variáveis estudadas, tais como velocidade de rotação dos discos (Figura 1a), teor de soluto (linalol) na alimentação (Figura 1b), razão entre as vazões mássica de solvente e alimentação (Figura 1c) e teor de água no solvente.
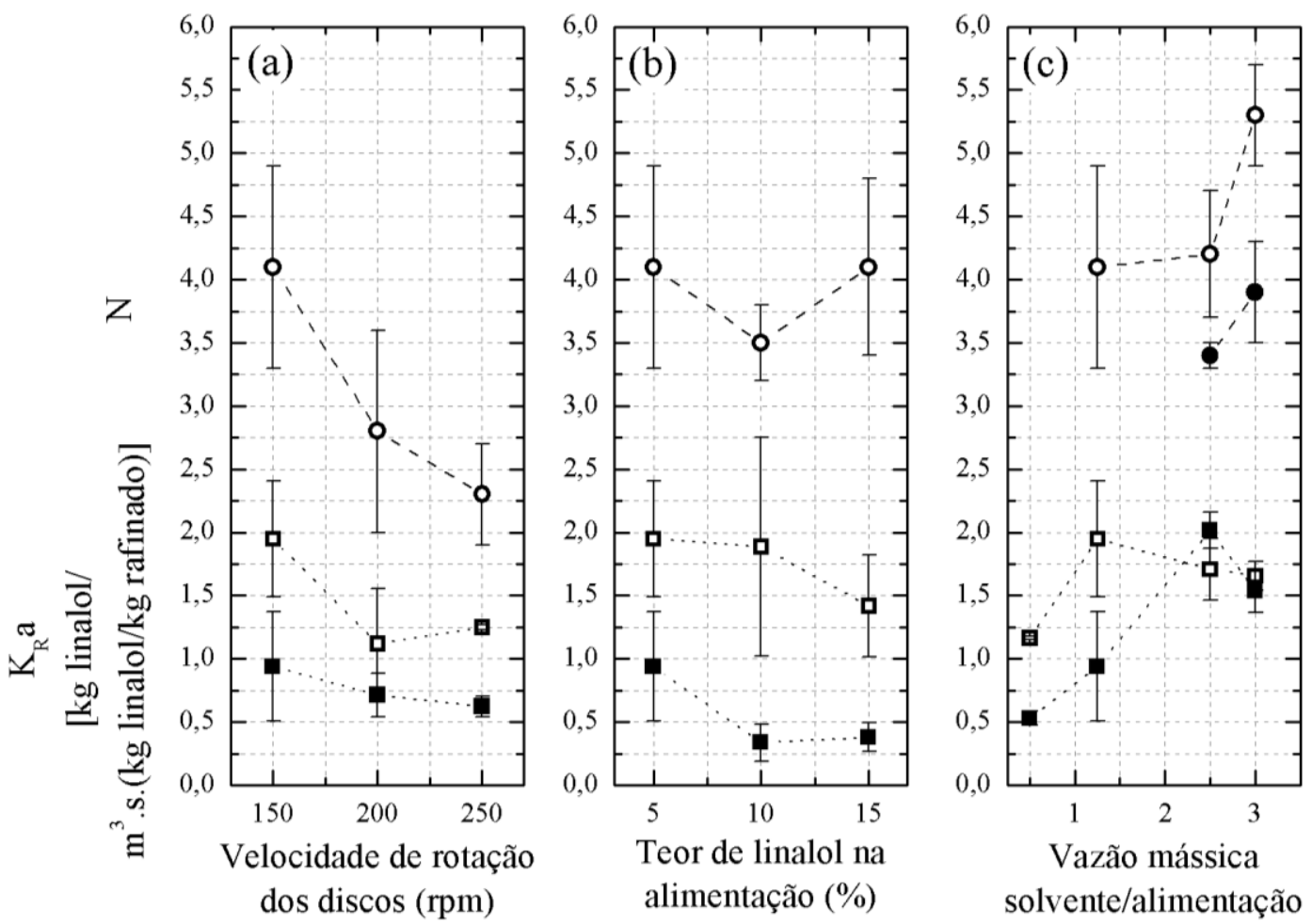

Figura 1 - Efeito das variáveis estudadas [(a) velocidade de rotação dos discos; (b) teor de linalol na alimentação; (c) razão entra as vazões mássicas de solvente e alimentação] sobre os valores

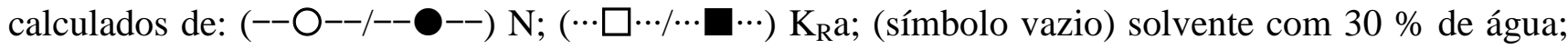
(símbolo cheio) solvente com $40 \%$ de água. Valores nominais de água expressos em massa. 


\section{9 a 22 de outubro de 2014 \\ Florianópolis/SC}

Como pode ser observado na Figura 1, em geral, os valores de $\mathrm{K}_{\mathrm{R}} \mathrm{a}$ variaram pouco com aumento de velocidade de rotação dos discos e com o aumento do teor de linalol na alimentação, ocorrendo uma maior mudança com o aumento da razão S/F a partir de 1,25. Provavelmente, o aumento da velocidade de rotação dos discos não exerceu muita influência na extração de linalol (Figura 1a), acarretando em uma diminuição nos valores de $\mathrm{K}_{\mathrm{R}} \mathrm{a}$ a partir de $200 \mathrm{rpm}$. $\mathrm{O}$ aumento do teor de linalol na mistura pode ter ocasionado aumento da solubilidade dos componentes e consequente diminuição ou pouca mudança na transferência mássica de linalol da fase rafinado para a fase extrato (Figura 1b).

Um aumento dos valores de $\mathrm{K}_{\mathrm{R}}$ a com o aumento da razão $\mathrm{S} / \mathrm{F}$ pode ser devido ao aumento da extração de linalol pela maior quantidade de solvente presente no equipamento (Figura 1c). Segundo descrito por Zuniga et al. (2006), o aumento da vazão do solvente em relação à vazão da alimentação favorece a troca mássica, uma vez que seu aumento acarreta na maior quantidade de linalol extraído. Entretanto, o aumento da quantidade de solvente em relação à alimentação também causa aumento da extração de limoneno. Este fato faz com que a transferência mássica de linalol seja prejudicada, acarretando em uma pequena diminuição dos valores de $\mathrm{K}_{\mathrm{R}} \mathrm{a}$.

Em relação ao teor de água no solvente, é possível observar que, na maioria dos casos, seu aumento acarreta na diminuição dos valores de $\mathrm{K}_{\mathrm{R}} \mathrm{a}$, possivelmente devido à menor extração de linalol. Para razões $\mathrm{S} / \mathrm{F} \geq 2,5$ os valores de $\mathrm{K}_{\mathrm{R}}$ a calculados para o solvente com $40 \%$ de água se igualam aos valores calculados para o solvente com $30 \%$ de água (Figura 1c).

Em relação aos valores do número de estágios do equipamento PRDC, em geral, seus valores são inversamente proporcionais aos valores de $\mathrm{K}_{\mathrm{R}} \mathrm{a}$, ou seja, quanto maior a transferência mássica do soluto (linalol), menor é o número de estágios calculados no equipamento (TREYBAL, 1963; 1980). Porém, muito pouco desse fenômeno pode ser percebido devido às grandes variações nos valores calculados para esta variável.

Em muito casos, não foi possível determinar valores de $\mathrm{N}$, sendo possível o cálculo para os experimentos com solvente com $40 \%$ de água somente a partir da razão $\mathrm{S} / \mathrm{F}=2,5$ e para o solvente com $30 \%$ de água a partir da razão $\mathrm{S} / \mathrm{F}=1,25$ (Figura 1c). Segundo descrito por Geankoplis (2003), quando a vazão do solvente $(\mathrm{S})$ é muito baixa, um caso limite é atingido. Neste caso, um número infinito de estágios será necessário para se realizar a operação desejada, tornando impossível seu cálculo. Sendo assim, denomina-se esta vazão de solvente como sendo a vazão mínima de solvente, sendo que vazões menores tornam o cálculo de $\mathrm{N}$ indeterminado. Desta forma, pode-se sugerir que a razão S/F mínima utilizando o solvente com $40 \%$ de água para que ocorra troca mássica no equipamento é de 2,5, e que a razão S/F mínima utilizando o solvente com $30 \%$ de água é igual a 1,25 (valores nominais).

De uma forma geral, observa-se que em praticamente todas as condições, os valores de $\mathrm{K}_{\mathrm{R}}$ a e $\mathrm{N}$ não variaram de forma expressiva, devido aos altos desvios calculados. O cálculo de $\mathrm{K}_{\mathrm{R}} \mathrm{a}$ e $\mathrm{N}$ tratamse de estimativas nas quais algumas considerações devem ser feitas atribuindo alguns erros aos cálculos, além de erros experimentais e analíticos. 


\section{9 a 22 de outubro de 2014 \\ Florianópolis/SC}

\section{CONCLUSÕES}

Os valores dos coeficientes globais volumétricos de transferência de massa de linalol $\left(\mathrm{K}_{\mathrm{R}} \mathrm{a}\right)$ não foram expressivamente afetados pelas condições monitoradas nos experimentos; em contrapartida, o número de estágios teóricos do equipamento PRDC sofreu diminuição com o aumento da velocidade de rotação dos discos e aumento com o aumento da razão solvente/alimentação. Também foi possível observar que o coeficiente global volumétrico de transferência de massa e o número de estágios teóricos diminuiram com o aumento da hidratação do solvente. Por fim, pode-se inferir que a utilização de razões $\mathrm{S} / \mathrm{F}$ menores que 2,5 para o solvente com $40 \%$ de água e $\mathrm{S} / \mathrm{F}$ menores que 0,5 para o solvente com $30 \%$ de água não é viável devido estas acarretarem no cálculo de um número infinito de estágios teóricos. De fato, os valores de razões $\mathrm{S} / \mathrm{F}$ supracitados são referentes às vazões mínimas de solvente.

\section{AGRADECIMENTOS}

Os autores gostariam de agradecer à Fundação de Amparo à Pesquisa do Estado de São Paulo (FAPESP, processos 2011/02476-7, 2013/11150-3 e 2010/13286-1) pelo apoio financeiro.

\section{REFERÊNCIAS}

ARCE, A.; MARCHIARO, A.; MARTínEZ-AGEITOS, J. M.; SOTO, A. Citrus Essential Oil Deterpenation by Liquid-liquid Extraction, Can. J. Chem. Eng., v. 83, p. 366-370, 2005.

CHÁFER, A.; TORRE, J.; MUÑOZ, R.; BURGUET, M. C. Liquid-liquid Equilibria of the mixture linalool+ethanol+water at different temperatures. Fluid Phase Equilibri., v. 238, p.72-76, 2005.

GEANKOPLIS, C. J. Transport Processes and Separation Process Principles. $4^{\mathrm{a}}$ ed. Prentice Hall, Nova Jersey, 2003.

GONÇALVES, D. Estudo do Processo de Desterpenação de Óleos Essenciais Cítricos: Dados de Equilíbrio Líquido-líquido e Extração em Coluna de Discos Rotativos Perfurados. Dissertação de mestrado, 180 p. Departamento de Engenharia de Alimentos, Faculdade de Zootecnia e Engenharia de Alimentos - FZEA/USP, Pirassununga/SP, 2013.

GONÇALVES, D.; KOSHIMA, C. C.; NAKAMOTO, K. T.; UMEDA, T. K.; ARACAVA, K. K.; GONÇALVES, C. B.; RODRIGUES, C. E. C. Deterpenation of eucalyptus essential oil by liquid + liquid extraction: Phase equilibrium and physical properties for model systems at T = 298.2 K. $J$. Chem. Thermodyn., v. 69, p. 66 - 72, 2014.

LAWRENCE, B. M. Cap. 3: The Isolation of Aromatic Materials from Natural Plant Products. In: SILVA, K. T. A Manual on the Essential Oil Industry. United Nations Idustrial Development Organization, VIENNA, AUSTRIA, 1995. 
PINA, C. G. Desempenho de uma Coluna de Discos Rotativos na Desacidificação do Óleo de Milho. Tese de Doutorado, 115 p. Faculdade de Engenharia de Alimentos - Universidade Estadual de Campinas (UNICAMP), Campinas/SP, 2001.

SCHMIDT, E. Cap. 4: Production of Essential Oils. In: BASER, K. H. C.; BUCHBAUER, G. HandBook of Essential Oils, CRC Press, Boca Raton - Flórida, Estados Unidos, 2010.

TREYBAL, R.E. Liquid Extraction. $2^{\mathrm{a}}$ ed. McGraw-Hill Book Company, Nova Iorque, Estados Unidos, 1963.

TREYBAL, R. E. Mass Transfer Operations. $3^{\text {a }}$ ed. McGraw-Hill Book Company, Singapura, 1980.

ZUNIGA, A. D. G.; COIMBRA, J. S. R.; MINIM, L. A.; ROJAS, E. E. G. Dispersed phase hold-up in a Graesser raining bucket contactor using aqueous two-phase systems. J. Food Eng., v. 72, p. 302309, 2006. 